\title{
Student Understanding of Differentials in Introductory Physics
}

\author{
Nathaniel R. Amos and Andrew F. Heckler \\ Dept. of Physics, The Ohio State University, 191 West Woodruff Ave, Columbus, OH 43210
}

\begin{abstract}
Evidence suggests that a major obstacle to student success in the construction of physics integrals is an inability to formulate and interpret differentials and products involving differentials. In differentials training, we conducted a controlled experiment at the introductory level to assess the effects of electronic feedback and physical context. This between-students design featured pairs of similarly-styled training tasks that varied by physical context, either on paper without feedback or on a computer with electronic feedback. A post-test featuring all physical contexts and several transfer questions was given to all conditions. We found significant differences in post-test score among the various physical contexts. Also, training with electronic feedback was seen to outperform both Control and paper-based training without feedback.
\end{abstract}

PACS: $01.40 . \mathrm{Fk}$

\section{INTRODUCTION}

Integration, which motivates our study of differentials, is a mathematical technique often needed to solve problems in introductory university physics courses. Over several decades, research has given insight into how students conceive of and use integration as a technique in their physics classes. Orton showed that students are frequently capable of computing integrals but often lack conceptual understanding of their underlying meaning [1]. Fundamentally, the meaning of an integral can be understood as an infinite sum of differential quantities. This notion of accumulating infinitesimally small elements is powerful and flexible; however, Meredith and Marrongelle found that students often rely on recall of similar problems or variable dependence when determining the need for physics integration, which can lead to inaccuracies [2].

Nguyen and Rebello divided the process of successfully completing a physics integral problem into several steps, one of which was setting up the differential quantity to be accumulated [3]. Their interviews with students suggested that a lack of physical understanding of the differential quantity caused them to sometimes arbitrarily append it to the end of an integrand or simply ignore it altogether, thus changing the answer. They proposed instructive tutorials to help students understand the meaning of infinitesimals and the process of accumulating them.

Recently, studies have attempted to identify the underlying reasoning that may lead to these student difficulties. Using the resource and conceptual metaphor frameworks, $\mathrm{Hu}$ and Rebello analyzed think-aloud group work from students solving physics integrals to categorize the type of reasoning they use when working with differential quantities [4]. Though they did not claim that utilization of unproductive resources necessarily yields incorrect physics integrals, their findings appear to suggest that students who reason with differentials as tiny fragments of a whole object in an infinite sum frequently find success in a wide variety of contexts.

As part of this larger effort in the PER community to advance student comprehension of integration in physics, we sought to promote improved conceptual understanding of differential quantities by training students with similarlystyled scaffolding questions. In particular, we intended to determine the effect of electronic feedback and whether any of a variety of physical contexts proved more advantageous in physics differentials training.

If students can begin to use expert-like language and descriptions of differentials and differential products (e.g., $F d x$ or $v d t$ ) as described above, they may be better prepared to reason with differentials in physics integration problems. Research has demonstrated that Computer Based Instruction (CBI) yields generally positive effects, especially when paired with feedback [5]; as a result, during the course of our experiment, some students received immediate electronic feedback to their responses from a computer terminal. The efficacy of electronic feedback has been shown to depend on a variety of factors, including the knowledge to be learned, student confidence, and the type of feedback given. In the case of physics differentials, this was considered an empirical question: we compared the trained performances of students receiving electronic feedback with those of students who had roughly identical paper-based training but lacking feedback. A more detailed description of the experiment follows in the next section.

\section{METHODS}

To determine the effects of feedback and physical context on students' understanding of physics differentials, we provided short training exercises (on a computer with electronic feedback or on paper with no feedback, across different contexts) and we followed this with a paper posttest. For comparison, a Control group received no training. 
In total, 320 students participated in this study. All students were enrolled in the second semester of a calculusbased, introductory physics course at Ohio State University. Students received course credit for their participation or an alternative assignment if they declined the study. Over $90 \%$ of students enrolled in the course participated in PER investigations; 320 of those participants were randomly selected for this particular study. Each participant completed a variety of computer and/or paper tasks in a quiet testing room for no more than 55 minutes.

Experimental conditions received two training exercises. Training included "Student A vs. Student B" argument questions, in which the participant selects an explanation about physical differentials with which they most agree; dimensional analysis questions to identify the units of physical differentials; as well as open-ended questions in which the participant is asked to describe differences between finite interval quantities (e.g., $\Delta t$ ) and infinitesimal quantities (e.g., $d t$ ). Each training task gradually focused its questions around the notion that differentials are infinitesimal physical quantities, in accordance with the "small amount" resource described in the previous section. All training tasks were given in sequential pairs (see Table 1). Pairs were chosen for a variety of reasons, including alternating or maintaining independent variables, which provides information about students' abilities to generalize.

Each training task concentrated on a dependentindependent pair of physical variables. This concentration was referred to as a "context." Training contexts used in this study were as follows: "velocity vs. time" (VT), "acceleration vs. time" (AT), "force vs. position" (FX), "linear mass density vs. position" (MDX), and "pressure vs. volume" (PV). Electronic training contexts with feedback featured identical questions to the paper versions, and are denoted "velocity vs. time electronic" (VTE), "force vs. position electronic" (FXE), and "pressure vs. volume electronic" (PVE). Students in the electronic feedback groups were told if their answers were correct or incorrect, and they were given correct explanations of each question.

Eight experimental conditions were employed. Participants were randomly assigned to one of the experimental conditions shown in Table 1. The conditions were (VT, AT), (AT, FX), (VT, FX), (FX, MDX), (FX, PV), (VTE, FXE), (FXE, PVE), and Control. The Control group received no training contexts on paper or computer.

Following training, all conditions were given an unrelated interim task for about 10 to 15 minutes to avoid the possibility that performance was based only on working memory from training. Participants then received a posttest, which consisted of a multiple-choice question, followed by open-ended questions. The questions included all training contexts, plus a generic/abstract context (see Figure 1) and a linear charge density context on which none received training.

Post-test questions asked students to describe/interpret differential quantities, identify units, and produce equations

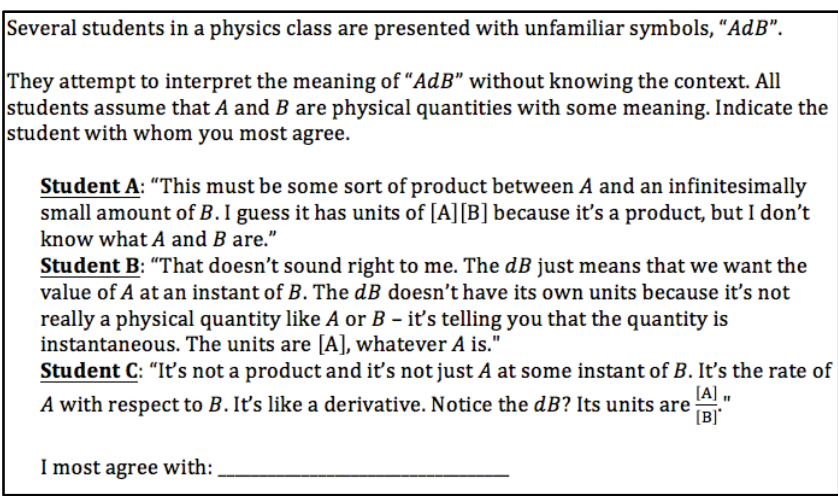

FIG 1. An example question from the differentials posttest. This multiple-choice, student-discussion question was given in a generic/abstract context.

relating differential quantities, such as $d x=v d t$. For posttest questions asking for interpretations of physical differentials, scoring emphasis was placed on descriptions/reasoning that appeared most consistent with the "small amount" resource. When calculating an overall post-test score, equal weight was given to each physical context as a whole to ensure that no training conditions were preferred. To further reduce bias towards any individual training condition, post-tests were scored before the results of the training exercises were examined.

TABLE 1. Experimental treatment design.

\begin{tabular}{ccc} 
Condition & Training Task 1 & Training Task 2 \\
\hline 1 & VT: No Feedback & AT: No Feedback \\
2 & AT: No Feedback & FX: No Feedback \\
3 & VT: No Feedback & FX: No Feedback \\
4 & FX: No Feedback & MDX: No Feedback \\
5 & FX: No Feedback & PV: No Feedback \\
6 & VTE: Feedback & FXE: Feedback \\
7 & FXE: Feedback & PVE: Feedback \\
8 & Control: None & Control: None \\
\hline
\end{tabular}

\section{RESULTS \& DISCUSSION}

\section{A. Effect Of Training In Different Physical Contexts}

While we do not intend to discuss all results from this study due to its data-rich nature, we will summarize several important findings. One of the primary goal of this study was to determine if short, contextual training (with or without feedback) improved reasoning and interpretation of physical differentials in introductory physics students compared with no training, and in particular if any physical contexts proved more successful than others. A visual summary of these results can be seen in Figure 2. A oneway ANOVA indicated statistically significantly different post-test scores among experimental conditions $(F(7,292)=6.734, p<0.001)$. Tukey's HSD showed that both feedback conditions, (VTE, FXE) $(p<0.001, d=1.28)$ and (FXE, PVE) $(p=0.001, d=$ 
1.16), and two paper/no feedback conditions, (VT, AT) $(p=0.001, d=0.88)$ and (VT, FX) $(p=0.003, d=$ 0.81), outperformed Control on the post-test.

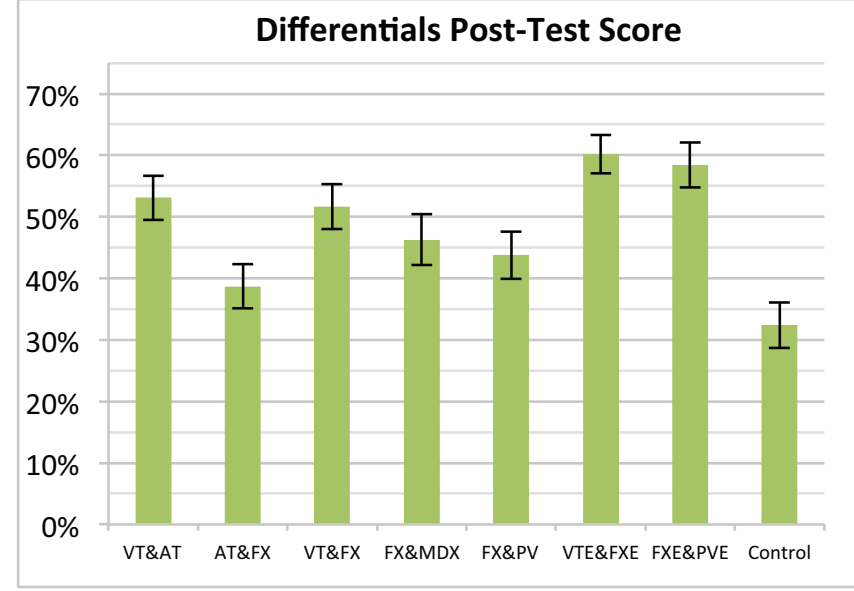

FIG 2. Mean differentials post-test scores (out of 100\%) for each experimental condition. Error bars are \pm 1 SE.

Our data also reveal that Control participants were more likely to describe differentials and differential products as "derivatives." This could be due to their unfamiliarity with the proper terminology. However, there is reason to believe that many students using language such as "rate" or "derivative" intend those meanings, as our results revealed numerous instances of students ascribing "rate-like" units to differential products, such as claiming that $v d t$ has units of $\mathrm{ms}^{-2}$. This interpretation was less common among the trained conditions than Control $\left(\chi^{2}(1, N=303)=\right.$ $18.1, p<0.001)$. When presented with a generic differential product devoid of physical meaning (as seen in Figure 1), 92\% (59 of 64) of participants who received computer training with feedback correctly interpreted it as a "product of $A$ with an infinitesimally small amount of $B$ " with units of $[A][B]$. On the same question, $66 \%$ (127 of 193 ) of students who received paper training without feedback also chose the correct answer. These results are contrasted with the $40 \%$ (17 of 43) of Control students who chose the answered correctly. Control students were more likely to choose the "derivative" interpretation (with "rate" units $[A][B]^{-1}$ shown) or a third possible answer, in which " $A d B$ " is evaluated "at an instant of $B$ " with units of $[A]$.

No training conditions explicitly prepared students for generic differential products. Because the post-test questions spanned all training contexts (and more) and students were explicitly trained on only two physical contexts per condition, most of the post-test questions can be regarded as contextual transfer. In Figure 3, we show the average post-test performances of each experimental condition partitioned according to their post-test training and transfer scores. "Transfer Contexts" (shown in red) represents the score (out of $100 \%$ ) on only the portion of post-test questions contextually unrelated to the condition's training tasks. Likewise, "Same Contexts" (shown in green) displays the score (out of $100 \%$ ) on only the portion of post-test questions belonging to the same physical context as training. Unsurprisingly, most conditions scored higher on questions with physical contexts from their training. In black, we compare the results of Control students on the same questions scored in green or red. Most training conditions (but not all) scored statistically significantly better than Control on the same set of questions, especially questions with contexts identical to their training tasks.

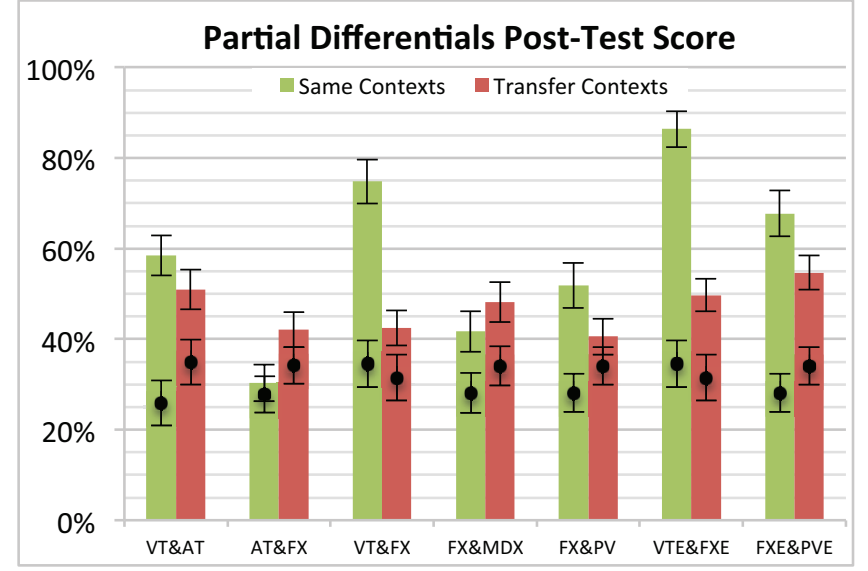

FIG 3. Mean differentials post-test scores for each condition, separated according to questions of same context as training tasks (green) and questions of different context as training tasks (red). Black circles indicate the performance of the Control condition on the same questions. Error bars are $\pm 1 \mathrm{SE}$.

Furthermore, a one-way ANOVA showed no significant differences between "Transfer Contexts" scores at the $\alpha=0.05$ level. It is possible that familiarity with some contexts from coursework could be expected to produce differences in "Transfer Contexts" scores, but this result remains under investigation. By contrast, visible differences in the green "Same Contexts" post-test scores are seen in Figure 3. The familiarity-hypothesis may explain our observation of relatively high "Same Contexts" scores in conditions featuring "velocity vs. time" compared to most other training conditions without (VT) training. However, this may also simply be an artifact of the post-test structure and still warrants further exploration.

\section{B. Effects Of Feedback And Course Grade}

Comparing the two training formats (computer/feedback and paper/no feedback) within the same two contextual combinations (VT\&FX; FX\&PV), a two-way ANOVA (feedback $x$ context) revealed a significant main effect of feedback $\quad(F(1,138)=10.086, p=0.002)$ and an insignificant main effect of training contexts $(F(1,138)=$ $1.772, p=0.185)$. Further, Tukey HSD tests found that feedback conditions outperformed same-context paper/no feedback training conditions $(p=0.008, d=0.55)$ and the Control condition $(p<0.001, d=1.27)$. These data suggest that electronic feedback can significantly enhance 
student understanding of physics differentials beyond paper training (without feedback) alone.

We also found evidence that electronic feedback may reduce the performance gap between stronger and weaker physics students. We examined the numbers of students in Low-Grade and High-Grade categories (split by median final physics course grade) and the numbers of students in Low-Score and High-Score differentials post-test categories (split by median post-test score). When separated by course grade, the performance gap (seen in conditions without feedback) diminishes with feedback.

High-Grade students in paper/no feedback training conditions were $60 \%$ (54 of 90) likely to also be HighScore students on the differentials post-test. However, LowGrade students in paper/no feedback training conditions were only $34 \%$ (33 of 97) likely to achieve the same result. By contrast, computer/feedback-trained conditions produced more equitable results between Low-Grade and High-Grade categories. Low-Grade, computer-trained students obtained high differentials post-test scores at a rate of $70 \%$ (21 of 30), which was identical to High-Grade, computer-trained students, 70\% (23 of 33) of whom also scored above the median on the post-test. We display the average post-test scores in each category in Figure 4.

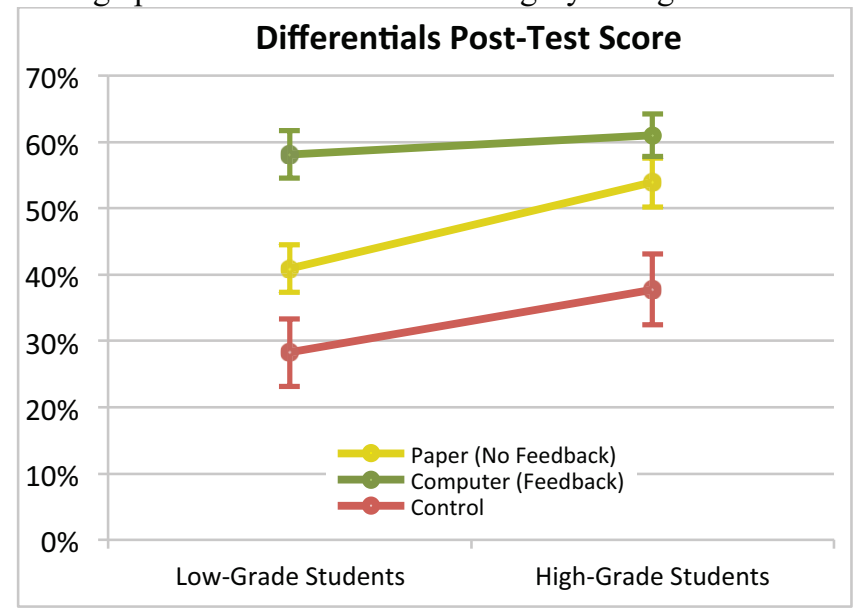

FIG 4. Mean differentials post-test scores separated by Low- and High-Grade students for each training format. Error bars are $\pm 1 \mathrm{SE}$.

According to Figure 4, the difference in post-test scores between Low- and High-Grade students is negligible when given feedback. Contrastingly, in same-context conditions without feedback, an independent-samples $t$-test found significantly higher post-test scores among High-Grade students than their Low-Grade peers $(t(75)=2.470, p=$ $0.016, d=0.57$ ).

[1] A. Orton, Educ. Stud. Math. 14, 1-18 (1983).

[2] D. Meredith and K. Marrongelle, Am. J. Phys. 76, (2008)

[3] D. H. Nguyen and N. S. Rebello, Phys. Rev. ST Phys. Educ. Res. 7, 010112 (2011).
It should be noted that an ANOVA revealed an insignificant (at the $\alpha=0.05$ level) interaction effect between course grade and feedback. Nevertheless, we can see that paperbased training does endow some benefit on both LowGrade and High-Grade students compared to untrained students of the Control condition, which produced the lowest average post-test scores across both grade categories. Indeed, a Tukey HSD test showed that paperbased training as a whole outperformed Control $(p<$ $0.001, d=0.62$ ), which suggests that some improvement in differentials understanding occurred even in the absence of feedback.

\section{CONCLUSION}

Overall the training resulted in some improvements on a short conceptual test of physical differentials, but the efficacy of the training was contingent on several factors. We found that electronic training with feedback and, to a lesser extent, identical paper-based training without feedback can promote improved descriptions, interpretations, and novel creations of physical differentials compared to Control. It may be argued that students can easily learn to mimic feedback without necessarily conceptualizing the meaning, which could result in artificially enhanced post-test scores when compared with untrained Control students. However, an expert-like description of a differential quantity is a necessary prerequisite to a richer demonstration of comprehension, and this alone is an improvement over most untrained responses to post-test questions.

In addition, we note that physical context seems to play a role in the benefit of this differentials training, where some contexts produce overall better results than others, which may be due to students' familiarity with certain physical scenarios/variables from their coursework. Furthermore, electronic feedback may narrow the performance gap between Low-Grade and High-Grade students in differentials, though paper-based training did confer some benefit to both groups over Control.

Finally, this study may have implications for the teaching of differentials in physics. Electronic and paperbased tutorials may improve students' descriptions, interpretations, and reasoning of physical differentials in only a short time period. This training could be given in small, recitation classes overseen by a TA (to provide feedback) or in an isolated environment without feedback. In both scenarios, conceptual gains may be evident among Low- and high-performing physics students.

[4] D. Hu and N. S. Rebello Phys. Rev. ST Phys. Educ. Res. 9, 020108 (2013)

[5] J. Hattie and H. Timperley, Rev. of Edu. Res. 77, (2007) 